\title{
Erratum to: Organizational Implementation of Evidence-Based Substance Abuse Treatment in Racial and Ethnic Minority Communities
}

\author{
Erick G. Guerrero • Amy He • Ahraemi Kim • \\ Gregory A. Aarons
}

Published online: 3 December 2013

(c) Springer Science+Business Media New York 2013

\section{Erratum to: Adm Policy Ment Health \\ DOI 10.1007/s10488-013-0515-3}

Unfortunately, in the original publication of the article, the narrative and Tables 3 and 4 presented the exponentiated regression coefficient estimates potentially confusing readers. Although all findings are unchanged in terms of statistical significance, we present here the narrative and corrected Tables 3 and 4 reporting the raw Beta estimates and standard errors.

1. The incorrect values should be replaced in the last line of the heading "Outer Context Hypothesis" under the "Results" section, on page 6 .

The correct sentence should read as:

Programs accepting private insurance were more likely to offer both CMT (B $=0.54, S E=0.23, p<0.05)$ and MAT (B $=0.47, S E=0.24, p<0.05$ ).

2. The incorrect values should be replaced in the last three sentences of second paragraph of the heading "Inner Context Hypotheses" under the "Results" section, on page 6.
The correct sentences should read as:

Supervisor attitudes toward EBPs, specifically openness (B $=0.37, S E=0.15, p<0.05)$ and regulation $(\mathrm{B}=0.28$, $S E=0.14, p<0.05)$, were statistically significantly related to CMT implementation. In addition, the most robust statistically significant relationship was found between supervisor readiness-for-change attributes and CMT (B $=0.78, S E=0.28, p<0.01)$. Supervisors' openness towards EBPs was also associated with MAT $(B=0.27$, $S E=0.14, p<0.05)$.

3. The incorrect values should be replaced in the last sentence of the third paragraph of the heading "Inner Context Hypotheses" under the "Results" section, on page 6.

The correct sentence should read as:

The interaction effect between private insurance and openness to EBPs was statistically significant $(\mathrm{B}=1.52, S E=0.56$, $p<0.01)$, as well as the interaction of parent organization and openness to EBPs $(\mathrm{B}=1.17, \mathrm{SE}=0.53, p<0.05)$.

Revised Tables 3 and 4 are presented below.

The online version of the original article can be found under doi: 10 . 1007/s10488-013-0515-3.

E. G. Guerrero $(\bowtie) \cdot$ A. He $\cdot$ A. Kim

School of Social Work, University of Southern California, 655 West 34th Street, Los Angeles, CA 90089-041, USA

e-mail: erickgue@usc.edu

A. $\mathrm{He}$

e-mail: ahe@usc.edu

A. Kim

e-mail: ahraemik@usc.edu

G. A. Aarons

Department of Psychiatry, University of California,

San Diego, San Diego, CA 92093, USA

e-mail: gaarons@ucsd.edu 
Table 3 Implementation of CMT $(N=122)$

Multivariate regression parameter estimates with robust standard errors from two-tailed tests

$C M T$ contingency management treatment, $T J C$ the joint commission

${ }^{\dagger} p<0.10 ; * p<0.05 ; * * p<$ 0.01

\begin{tabular}{|c|c|c|c|c|c|c|}
\hline \multirow[t]{3}{*}{ Variable } & \multicolumn{6}{|c|}{ Implementation of CMT } \\
\hline & \multicolumn{2}{|l|}{ Model 1} & \multicolumn{2}{|l|}{ Model 2} & \multicolumn{2}{|l|}{ Model 3} \\
\hline & $\mathrm{B}$ & $S E$ & B & $S E$ & B & $S E$ \\
\hline \multicolumn{7}{|l|}{ Organization } \\
\hline State licensure & 0.07 & 0.59 & 0.13 & 0.64 & 0.09 & 0.51 \\
\hline TJC accreditation & $0.68^{*}$ & 0.33 & $0.62 *$ & 0.34 & $0.51^{\dagger}$ & 0.30 \\
\hline Public funding & -0.19 & 0.30 & -0.17 & 0.32 & -0.22 & 0.28 \\
\hline Parent organization & -0.34 & 0.23 & -0.28 & 0.24 & $-0.45^{*}$ & 0.22 \\
\hline Medicaid & $-0.46^{\dagger}$ & 0.25 & $0.49^{\dagger}$ & 0.26 & -0.23 & 0.24 \\
\hline Private insurance & $0.52 *$ & 0.24 & $0.59 *$ & 0.25 & $0.54 *$ & 0.23 \\
\hline Private insurance $\times$ openness to EBPs & & & & & -0.26 & 0.72 \\
\hline Parent organization $\times$ openness to EBPs & & & & & -0.37 & 0.62 \\
\hline \multicolumn{7}{|l|}{ Director and staff } \\
\hline Director leadership & & & $0.23^{\dagger}$ & 0.13 & $0.18^{\dagger}$ & 0.11 \\
\hline Staff resources for change & & & 0.06 & 0.24 & 0.02 & 0.22 \\
\hline Staff education & & & -0.41 & 0.36 & -0.23 & 0.33 \\
\hline \multicolumn{7}{|l|}{ Clinical supervisor } \\
\hline Field tenure & & & & & $0.02^{\dagger}$ & 0.01 \\
\hline Education & & & & & -0.03 & 0.07 \\
\hline \multicolumn{7}{|l|}{ Attitudes toward EBP } \\
\hline Openness & & & & & $0.37 *$ & 0.15 \\
\hline Regulation & & & & & $0.28 *$ & 0.14 \\
\hline Appeal & & & & & -0.12 & 0.15 \\
\hline Divergence & & & & & -0.26 & 0.19 \\
\hline Attributes for change & & & & & $0.78^{* *}$ & 0.28 \\
\hline Constant & $3.48 * *$ & 0.61 & $2.40^{*}$ & 1.10 & -1.83 & 1.48 \\
\hline Adjusted $R^{2}$ & 0.17 & & 0.21 & & 0.39 & \\
\hline
\end{tabular}


Table 4 Implementation of $\operatorname{MAT}(N=122)$

Multivariate regression parameter estimates with robust standard errors from two-tailed tests

MAT medication assisted treatment, $T J C$ the joint commission

${ }^{\dagger} p<0.10 ; * p<0.05 ; * * p<$ 0.01

\begin{tabular}{|c|c|c|c|c|c|c|}
\hline \multirow[t]{3}{*}{ Variable } & \multicolumn{6}{|c|}{ Implementation of MAT } \\
\hline & \multicolumn{2}{|l|}{ Model 1} & \multicolumn{2}{|l|}{ Model 2} & \multicolumn{2}{|l|}{ Model 3} \\
\hline & B & $S E$ & $\mathrm{~B}$ & $S E$ & B & $S E$ \\
\hline \multicolumn{7}{|l|}{ Organization } \\
\hline State licensure & 0.22 & 0.24 & 0.24 & 0.25 & 0.42 & 0.32 \\
\hline TJC accreditation & 0.40 & 0.41 & 0.39 & 0.36 & 0.46 & 0.34 \\
\hline Public funding & -0.51 & 0.32 & $-0.57^{\dagger}$ & 0.34 & -0.48 & 0.32 \\
\hline Parent organization & $0.45^{\dagger}$ & 0.25 & 0.29 & 0.26 & 0.19 & 0.27 \\
\hline Medicaid & $0.40^{\dagger}$ & 0.22 & $0.43^{\dagger}$ & 0.22 & $0.38^{\dagger}$ & 0.20 \\
\hline Private insurance & 0.35 & 0.27 & $0.43^{*}$ & 0.26 & $0.47 *$ & 0.24 \\
\hline Private insurance $\times$ openness to EBPs & & & & & $1.52 * *$ & 0.56 \\
\hline Parent organization $\times$ openness to EBPs & & & & & $1.17 *$ & 0.53 \\
\hline \multicolumn{7}{|l|}{ Director and staff } \\
\hline Director leadership & & & -0.03 & 0.14 & -0.04 & 0.14 \\
\hline Staff resources for change & & & 0.18 & 0.22 & 0.25 & 0.21 \\
\hline Staff education & & & 0.46 & 0.33 & 0.52 & 0.33 \\
\hline \multicolumn{7}{|l|}{ Clinical supervisor } \\
\hline Field tenure & & & & & 0.02 & 0.02 \\
\hline Education & & & & & 0.12 & 0.08 \\
\hline \multicolumn{7}{|l|}{ Attitudes toward EBP } \\
\hline Openness & & & & & $0.27 *$ & 0.14 \\
\hline Regulation & & & & & -0.17 & 0.13 \\
\hline Appeal & & & & & 0.12 & 0.15 \\
\hline Divergence & & & & & 0.13 & 0.19 \\
\hline Attributes for change & & & & & -0.05 & 0.29 \\
\hline Constant & $1.25 * *$ & 0.35 & 0.65 & 0.82 & -1.37 & 1.47 \\
\hline Adjusted $R^{2}$ & 0.13 & & 0.16 & & 0.32 & \\
\hline
\end{tabular}

